\title{
Flexible Hinge Parameter Design and Performance Simulation of Vibration Assisted Machining Platform
}

\author{
Yixiong Yan $^{1}$ \\ ${ }^{1}$ Central South University, Mechanical and Electrical Engineering Department,410083Changsha Hunan, China
}

\begin{abstract}
Vibration assist machining is a modern mechanical manufacturing technology which is aimed at achieving an efficient high-quality machining. This article focuses on a new-type vibration assisted machining platform which based on the stiffness and frequency requirement to obtain the key structural parameter and nature frequency of the platform by using the finite element method. Subsequently, static strength of the platform has been analyzed. Moreover, different excitation frequencies of the piezoelectric ceramics have been loaded at two vertical directions. The trajectory stability and characteristics of the platform under different actuating frequency have been figured out by using the finite element multi-body contact transient response analysis method. The results show that the nature frequency excitation results in large and destructive displacement. The steady-state response trajectory of the platform is elliptical under different excitation frequencies while under high excitation frequency it would become an elliptical moving. Once the unstable vibration time of platform increase, the unstable vibration amplitude would increase. The research results can provide a basis for the development of a new-type vibration assisted machining platform and the processing technology.
\end{abstract}

\section{Introduction}

Vibration assisted machining has become one of the most important technologies for precision and ultra-precision machining. During processing, it is advantageous to have controlled vibration with high frequency and low amplitude [1]. Surface and subsurface damage would take place due to the brittle fracture during the traditional process [2]. The vibration assisted machining can reduce machining force in the process, resulting in an increasing of machining surface accuracy and reduction of tool wear [3]. In recent years, vibration assisted machining technology has extensive applications in ultrasonic vibration cutting, grinding, plastic processing with its rapid development. This technology also solves a considerable number of problems in relation to difficult-to-process materials machining. It can be predicted that the application would be more and more widely [4].

The vibration assisted machining platform is the key component to achieve vibration assisted cutting. In order to meet the requirements of high efficiency and high-quality cutting, this paper designs the key structure of the flexible hinges which functions as the transmission component of the platform. Moreover, the dynamic and static performance of the platform have been analysed by using the finite element analysis simulation. It lay the foundation for the development of a new type of vibration assisted platform.

\section{Structure and components of the vibration assisted platform}

Vibration assisted machining platform is used for cutting, grinding and other processing to improve the processing technology [5]. The platform mainly consists of piezoelectric ceramic placement slots, flexible hinges and a stage. The structure is shown in Figure 1.

In order to ensure that the flexible hinges work without cross-coupling displacement and the vibration can be smoothly conducted in both directions, the structure of the flexible hinges is designed as a double-layer structure. The inner layer hinges guide the vertical vibration while the outer layer hinges guide the horizontal vibration.

The material used for the platform is $30 \mathrm{CrMnSiA}$ alloy structural steel, its material performance parameters are shown in Table 1.

The actuation components are piezoelectric ceramics, mounted in two perpendicular directions [manufactured by Physiklnstrumente (PI) GmbH \& Co.KG, Germany]. According to their model parameters and platform quality parameters, the stiffness of the platform and the limitation of natural frequency are obtained. Stiffness should be less than $30 \mathrm{~N} / \mathrm{um}$, while the natural frequency should be greater than $2500 \mathrm{~Hz}$. 


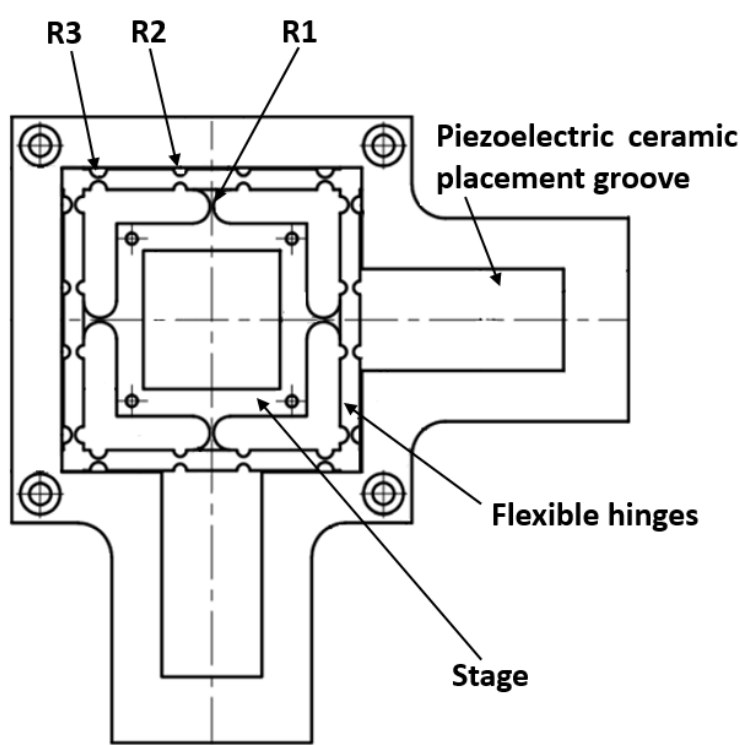

Figure 1. The structure of the platform

Table 1. Carbon structural steel performance parameters

\begin{tabular}{ccc}
\hline Property & Value & Unit \\
\hline Density & 7850 & $\mathrm{~kg} / \mathrm{m}^{3}$ \\
Young's Modulus & $1.96 \mathrm{E}+11$ & $\mathrm{~Pa}$ \\
Poisson's Ratio & 0.3 & - \\
Bulk Modulus & $1.6333 \mathrm{E}+11$ & $\mathrm{~Pa}$ \\
Shear Modulus & $7.5385 \mathrm{E}+10$ & $\mathrm{~Pa}$ \\
\hline
\end{tabular}

\section{Structural parameters of flexible hinges}

\subsection{Flexible hinges components structure analysis}

Figure 2 shows the structure of the flexible hinge, where $b$ is the width of the flexible hinge, $t$ is the minimum thickness of the middle part of it, $\mathrm{R}$ is the radius of the notch, $\mathrm{L}$ is the length of it, the rotational stiffness on the z-axis and the tensile stiffness on the x-axis is $k_{\alpha_{z}}$ and $k_{x}$, respectively. The minimum thickness $t$ and the width $b$ are fixed values, the angular deformation $\triangle \mathrm{x}$ can be expressed by the stiffness equation (1) [6].

$$
k_{s}=\frac{F_{x}}{\Delta x}=\frac{E b}{\left[\frac{2(2 s+1)}{4 s+1} \arctan \sqrt{4 s+1}-\frac{\pi}{2}\right]}
$$

Where $s=R / t$. It can be seen that the stiffness is mainly affected by the cutting radius $R$. And the flexible hinge component is symmetrical as shown in Figure 1, due to the fact that flexible hinge component is symmetrical, only three notch radii $\mathrm{R} 1, \mathrm{R} 2$ and $\mathrm{R} 3$ require to be considered which are marked in figure 1 .

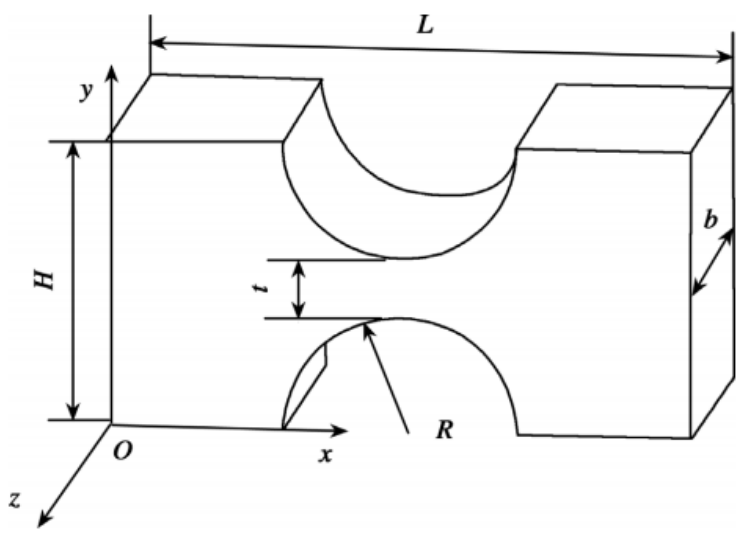

Figure 2. Structure of the flexible hinge

\subsection{Flexible hinge parameter design}

In order to figure out these three notch radii, several works should be done. First, a clear and definite goal should be defined as it is the basis, followed by the building of a mathematical model. With this model, ANSYS Workbench is used to work out the parameters. The greater stiffness of the platform means that the piezoelectric ceramics need to provide greater driving force, which will increase the operating temperature. As a result of high operating temperature, the actuator performance will be degraded. Therefore, the stiffness of the platform should be as small as possible [7]. In order to widen the working frequency range of the platform and better avoid the resonance effect, the natural frequency of the platform should be as high as possible [8]. At the same time, the relationship between the stiffness and the natural frequency of the platform are expressed by equation (2).

$$
\omega_{0}=\frac{1}{2 \pi} \sqrt{\frac{k_{x}}{M}}
$$

It can be seen that when the mass of the platform is fixed, the value of the natural frequency and stiffness is proportional. In summary, the objective of the solution is to ensure the natural frequency of the platform is as large as possible and the stiffness is as small as possible under the condition of mutually restrictive.

The range of R1, R2, R3 according to the overall structure design decision are shown as equation (3).

$$
\left\{\begin{array}{c}
2<R 1<5 \\
0.5<R 2<2.9 \\
0.5<R 3<2.9
\end{array}\right.
$$

The mathematical model can be expressed as equation (4):

$$
\left\{\begin{array}{l}
\text { Max } f=f(R 1, R 2, R 3) \\
\text { Min } k=k(R 1, R 2, R 3)
\end{array}\right.
$$

After the mathematical model is built, the established platform model is imported into the ANSYS Workbench. The range of the notch radii is set as equation (3). Then the software generates random and eligible 2000 groups $\mathrm{R} 1, \mathrm{R} 2, \mathrm{R} 3$ to obtain the corresponding stiffness and 
natural frequency of the platform. The optimal result is presented in Table 2 .

Table 2. Parameter solving result

\begin{tabular}{ccccc}
\hline R1 & R2 & R3 & Stiffness & $\begin{array}{c}\text { Natural } \\
\text { frequency }\end{array}$ \\
\hline $4.95 \mathrm{~mm}$ & $1.14 \mathrm{~mm}$ & $1.71 \mathrm{~mm}$ & $27.7 \mathrm{~N} / \mathrm{um}$ & $2625 \mathrm{~Hz}$ \\
\hline
\end{tabular}

\section{Static performance of the platform}

The flexible hinges of the platform will be subjected to the dynamic force of the actuator during operation, and its strength must be able to withstand the peak thrust of the actuator.

The designed platform model is imported into ANSYS Workbench. Hexahedron-dominated method is selected as meshing method while approximate and curvature control method in the advanced dimension control set group is used to mesh the curve structure. The minimum division size is $0.16 \mathrm{~mm}$, and a total of 219493 units are obtained. The maximum working thrust is $2000 \mathrm{~N}$, which is applied to the platform, and the static analysis result is shown in Figure 3.

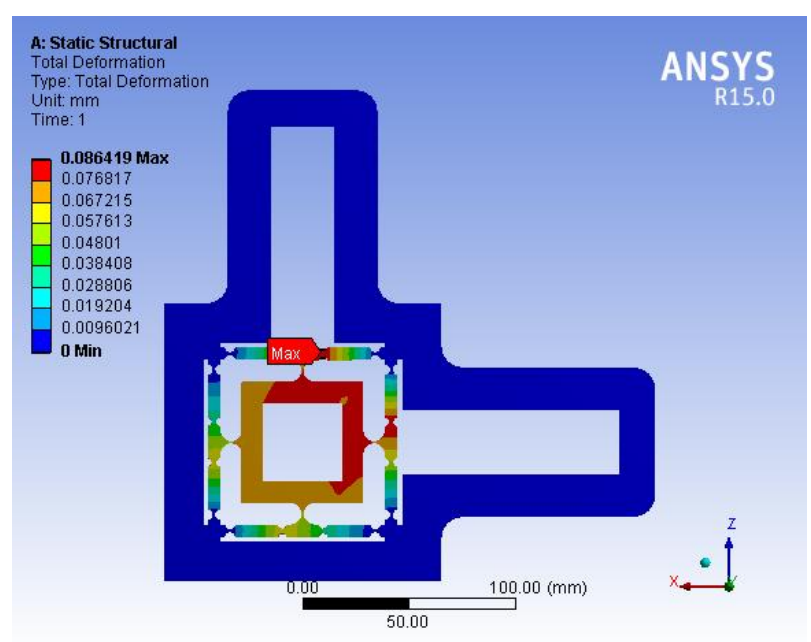

a) Deformation distribution

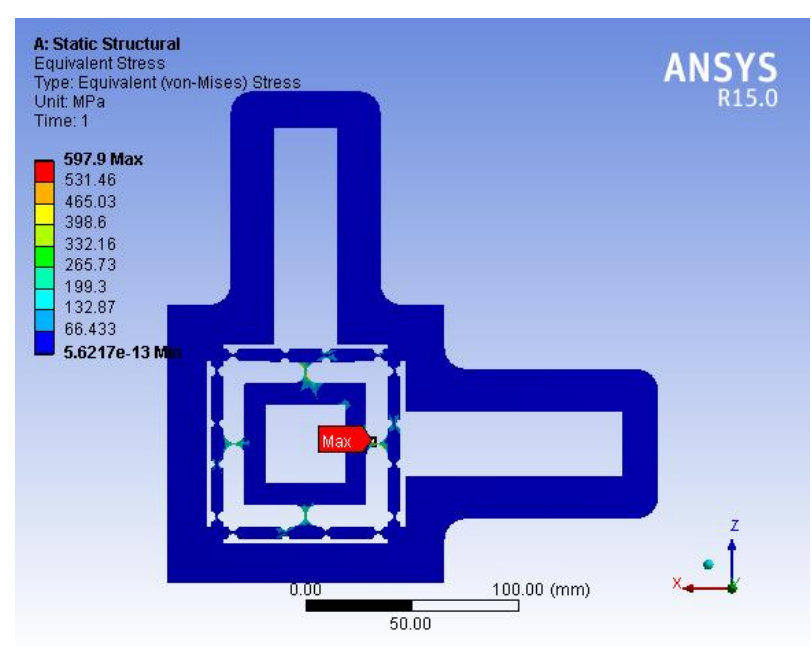

b) Stress distribution
Figure 3. Static performance of the platform

It can be seen from the simulation result that the maximum deformation of the platform is $0.086 \mathrm{~mm}$. The main deformation occurs at the flexible hinges, and the deformation is relatively reasonable. The maximum stress value is $597.9 \mathrm{Mpa}$ while the yield point of the material is $\sigma_{s}=1080 \mathrm{Mpa}$. Thus the safety factor is greater than 1.5 , so that the static performance of the platform meets the requirement.

\section{Transient response analysis of the platform}

\subsection{Transient response analysis}

The transient dynamics solution uses the modal superposition method to derive eigenvectors. By multiplying and adding the eigenvectors. The response of the structure can be solved [9]. Through the transient response analysis, the vibration rule of the platform under different external excitation can be obtained.

The platform model is imported into the ANSYS Workbench. Two vertical external excitations, generated by piezoelectric ceramics, are applied to the platform based on the model analysis. The mechanical equation of the excitations are expressed as follow.

$$
\begin{aligned}
& F=A \sin (2 \pi f t+\varphi) \\
& F=A \cos (2 \pi f t+\varphi)
\end{aligned}
$$

Considering that the natural frequency of the platform is $2625 \mathrm{~Hz}$, four groups of excitation frequencies are selected for simulation: $500 \mathrm{~Hz}, 2000 \mathrm{~Hz}, 2625 \mathrm{~Hz}$, $3000 \mathrm{~Hz}$. In accordance with the requirements of the platform, the amplitude value is set as $4 \mathrm{um}$. Four groups of results are shown as below.

\subsubsection{The transient response analysis result under $500 \mathrm{~Hz}$ excitation}

The transient response in the $\mathrm{X}$-axis direction of the reference point on the stage of the platform when the excitation frequency is $500 \mathrm{~Hz}$ is shown in Figure 4. The platform keeps unsteady vibration within 0.1 second while the maximum amplitude reaches $7.3329 \mathrm{E}-03 \mathrm{~mm}$. Moreover, the vibration began to stabilize when near 0.2 second. The deformation response trend in $\mathrm{Z}$-axis direction is very similar to the $\mathrm{X}$-axis.

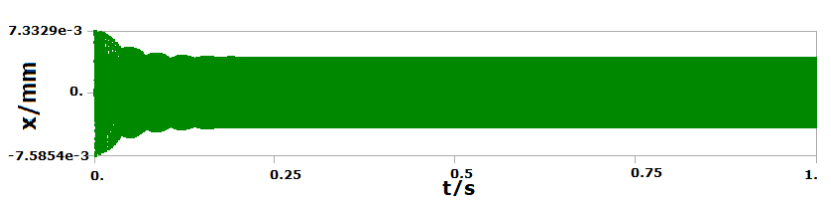

Figure 4. The deformation response in $\mathrm{X}$-axis direction when loading $500 \mathrm{~Hz}$ excitation 
According to the displacement data in $\mathrm{X}$ and $\mathrm{Z}$ direction, the trajectory chart of the reference point on the platform can be obtained in Figure 5. The 'wave' line refers to the trajectory in the unstable period, and the 'stable' line refers to the trajectory in the stable period. It can be seen that the trajectory in unstable period is similar to a rectangle, which transpires at the both ends of the $\mathrm{X}$-axis. While the trajectory in the stable period basically coincide as a smooth ellipse, and the long axis of the ellipse is at an about $45^{\circ}$ to the $\mathrm{X}$-axis.

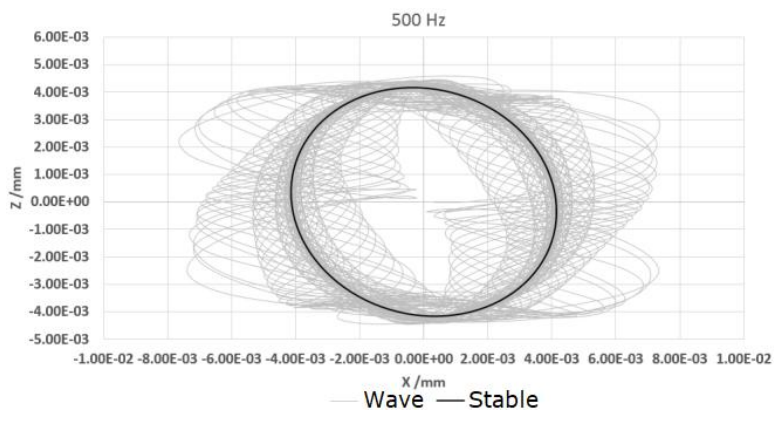

Figure 5. Trajectory chart of the deformation during $500 \mathrm{~Hz}$

\subsubsection{The transient response analysis result under $2000 \mathrm{~Hz}$ excitation}

The transient response in the $\mathrm{X}$-axis direction of the reference point on the stage of the platform when the excitation frequency is $2000 \mathrm{~Hz}$ is shown in Figure 6. The maximum amplitude of the unstable period is $2.009 \mathrm{E}-02 \mathrm{~mm}$. The amplitude is greater than before, and the unstable period lasts longer. However, the transition from unstable to stable vibration is quite smooth.

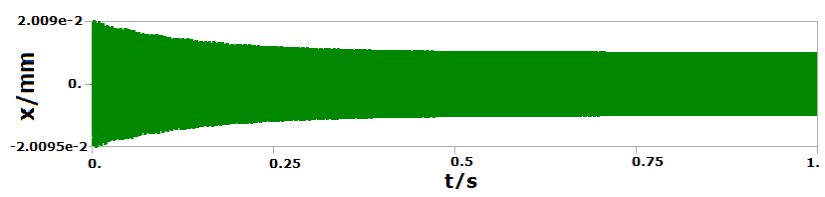

Figure 6. The deformation response in $\mathrm{X}$-axis direction when loading $2000 \mathrm{~Hz}$ excitation

The reference point displacement in $\mathrm{X}-\mathrm{Z}$ plane is shown in Figure 7. The unstable period trajectory is similar to an ellipse. However, the route trend is not obvious. The overlap rate of the unstable period trajectory is higher than before. The stable period trajectory coincide as a smooth ellipse while the axes of it are parallel to the $\mathrm{X}$-axis and $\mathrm{Z}$-axis.

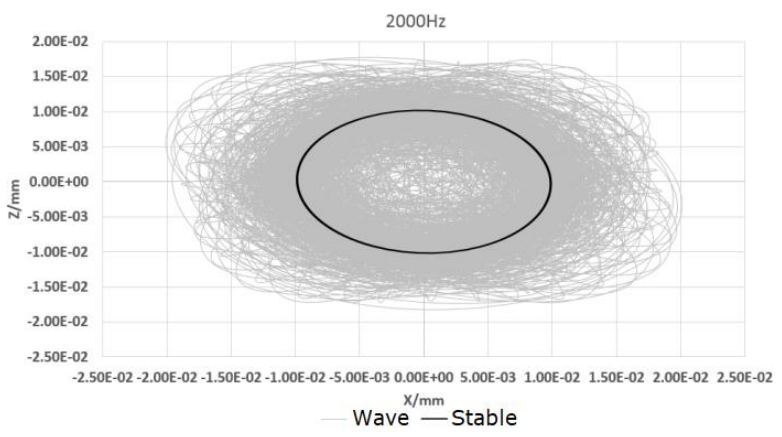

Figure 7 Trajectory chart of the deformation during $2000 \mathrm{~Hz}$

\subsubsection{The transient response analysis result under $2625 \mathrm{~Hz}$ (natural frequency) excitation}

The transient response in the $\mathrm{X}$-axis direction of the reference point on the stage of the platform when the excitation frequency is $2625 \mathrm{~Hz}$ is shown in Figure 8. The unstable period increased significantly, and the maximum amplitude reaches a value of $1.0061 \mathrm{~mm}$. The strong vibration may cause damage to the platform so that it is essential to avoid such circumstance.

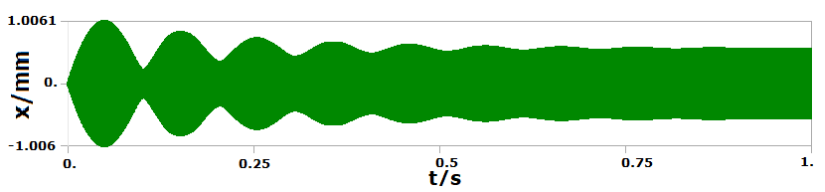

Figure 8. The deformation response in $\mathrm{X}$-axis direction when loading $2625 \mathrm{~Hz}$ excitation

The reference point displacement in $\mathrm{X}-\mathrm{Z}$ plane is shown in Figure 9. The route trend is quite clear even in unstable period.

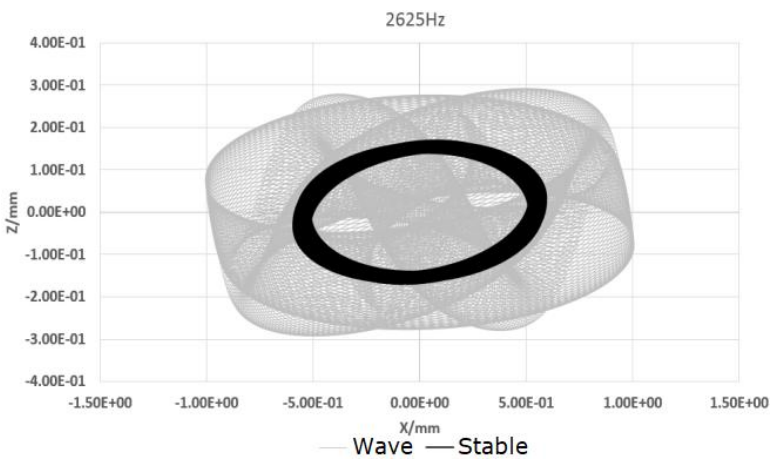

Figure 9. Trajectory chart of the deformation during $2625 \mathrm{~Hz}$

\subsubsection{The transient response analysis result under $3000 \mathrm{~Hz}$ excitation}

The transient response in the $\mathrm{X}$-axis direction of the reference point on the stage of the platform when the excitation frequency is $3000 \mathrm{~Hz}$ is shown in Figure 10. The unstable period is much shorter than last one, and the maximum amplitude of the unstable period is 2.7283E-02mm.

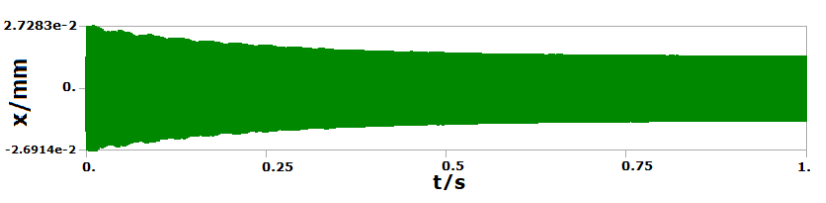

Figure 10 The deformation response in $\mathrm{X}$-axis direction when loading $3000 \mathrm{~Hz}$ excitation

The reference point displacement in $\mathrm{X}-\mathrm{Z}$ plane is shown as Figure 11. It can be seen that the unstable period route trend is unclear, but the unstable period is 
shorter than last one. The trajectory in stable period still coincide as a smooth ellipse.

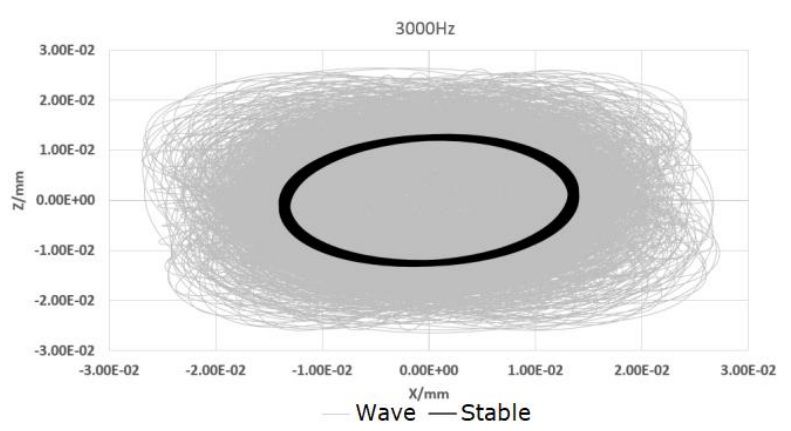

Figure 11. Trajectory chart of the deformation during $3000 \mathrm{~Hz}$

The response characteristics of the platform in different operating frequency can be conclude in the table below.

Table 3. Response characteristics at different excitation frequencies

\begin{tabular}{cccc}
\hline $\begin{array}{c}\text { Frequency } \\
\text { /Hz }\end{array}$ & $\begin{array}{c}\text { Duration in } \\
\text { unstable period } \\
\text { /s }\end{array}$ & $\begin{array}{l}\text { Maximum amplitude in } \\
\text { unstable period } \\
/ \mathbf{m m}\end{array}$ & $\begin{array}{l}\text { Amplitude in } \\
\text { stable } \\
\text { /mm }\end{array}$ \\
\hline 500 & 0.208 & $7.3 \mathrm{E}-03$ & $4.3 \mathrm{E}-03$ \\
2000 & 0.625 & $2.0 \mathrm{E}-02$ & $1.2 \mathrm{E}-02$ \\
2625 & 0.875 & 1.006 & 0.759 \\
3000 & 0.812 & $2.7 \mathrm{E}-02$ & $1.3 \mathrm{E}-02$ \\
\hline
\end{tabular}

\section{Conclusion}

1) With the aim of small stiffness and high natural frequency, the key parameters of the flexible hinges structure is obtained by the finite element method. 2) The static strength and deformation of the platform under the external excitation produced by piezoelectric ceramics are calculate and the reliability of the static strength of the platform is ensured. 3) The finite element method is used to analyze the transient response of the platform under different frequency excitations. Moreover, the response characteristics of the platform has been presented: The trajectory of the reference point on the platform in the stable period coincide as a smooth ellipse when two excitations loading in two vertical directions. Under the excitation of natural frequency, the duration of the unstable period is long, and the amplitude of the vibration is harmful to the platform. It provides a basis for choosing different excitation parameters for different processing technologies.

\section{Acknowledgements}

The authors express their deep gratitude to the National Natural Science Foundation of China (NSFC) (No. 51575533)

\section{References}

1. W. Xu and L. Zhang, AIM, 3, 173-192 (2015)

2. D.E. Brehl* and T. Dow, PE, 32, 153-172 (2008)

3. T. Tawakoli, B. Azarhoushang and M. Rabiey, TIJOAMT, 42(9/10), 883-891 (2008)

4. Z. Zhou, Q. Zhou and Y. Ren, JOME, 46(17), 105 (2010)

5. X. Zhang and F. Jiao, TE, 1, 3-8 (2012)

6. Z. Yu, X. Yao and X. Song, CJOSI, 30(9), 1818-1822 (2009)

7. B. Jaffe, W. Cook and H. Jaffe, JOSV, 20(4), 271-279 (1971)

8. H. Ji, and Y. Wen, AMR, 179-180, 1368-1373 (2011)

9. Q. Zhang, X. Chen, Z. Zhu, et al., 85(3), 1819 (2013) 